\title{
Determination of Punicalagin Isomers in Pomegranate Husk
}

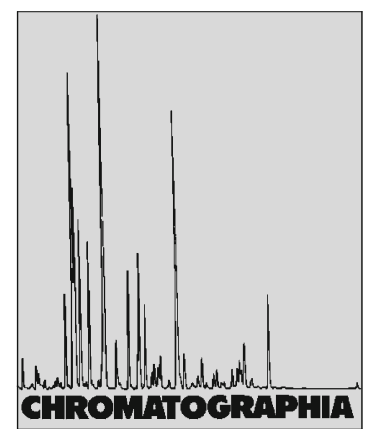

2008, 68, 303-306

\author{
Jingjing Lu ${ }^{1}$, Ke Ding ${ }^{2}$, Qipeng Yuan ${ }^{1, 凶}$ \\ ${ }^{1}$ Key Laboratory of Bioprocess of Beijing, Beijing University of Chemical Technology, Beijing 100029, China; \\ E-Mail: yuanqp@mail.buct.edu.cn \\ 2 Department of Food Science, Beiijing University of Agriculture, Beijing 102206, China
}

Received: 5 November 2007 / Revised: 4 May 2008 / Accepted: 5 May 2008

Online publication: 25 June 2008

\begin{abstract}
Punicalagins are the main ingredients of phenolic compounds in pomegranate (Punica granatum L.) husk. A simple and accurate method for punicalagin analysis based on ethanol extraction and RP-LC using linear gradient of methanol in $0.1 \%$ TFA solution was established. The feasibility of this procedure was tested by analyzing the punicalagin level both in fresh pomegranate husk collected from different provinces in China and dried husk from a drugstore. The content of each isomer and total content of punicalagins in husk were determined. The mean value of punicalagins content in pomegranate husk was $82.4 \mathrm{mg} \mathrm{g}^{-1}$. The highest content of punicalagins was found in a variety of husks from Shanxi province, while the lowest content was found in the husk from the drugstore in Guangdong province.
\end{abstract}

\section{Keywords}

Column liquid chromatography

Pomegranate husk

Punincalagin

\section{Introduction}

Pomegranate (Punica granatum L.) husk, a traditional Chinese medicine used as antibacterial, anti-inflammatory and hemostasis agent, is rich of phenolic compounds [1]. The most abundant of these polyphenols is punicalagin.
Nearly one-third of the provinces in China grow pomegranate trees. Punicalagin levels in pomegranate husk are of great difference depending on the area for fruit growth. At the same time, processing and storage conditions do influence the punicalagin content in dried husk.
Punicalagin is a potent antioxidant. The potent bioactivity of punicalagins can be explained by its ability to hydrolyze into ellagic acid (EA) in vivo and across the mitochondrial membrane in vitro. It was also found to protect against oxidative damages to lipids, amino acids and guanosine as [2].

Ellagic acid is a dietary polyphenol found in fruits and nuts and implicated with potent antioxidant, anticancer and antiatherosclerotic biological properties. Free EA in human plasma is due to its release from the hydrolysis of ellagitannins such as punicalagins [3].

Due to the importance of this compound, methods such as liquid chromatography (LC) [4-6] and LC-MS [7] have been used to analyze punicalagin in pomegranate extracts. Punicalagins are often noted in the singular punicalagin, however, it is found naturally as two reversible $\alpha$ - and $\beta$-anomers. Considering this aspect, punicalagins are difficult to analyze quantitatively.

Furthermore, all reported methods were described as time consuming and were incapable of screening a large number of samples without elaborate sample preparation. For example, they need purification by Sephadex-LH-20 [4] and sample preparation was by Toyo- 

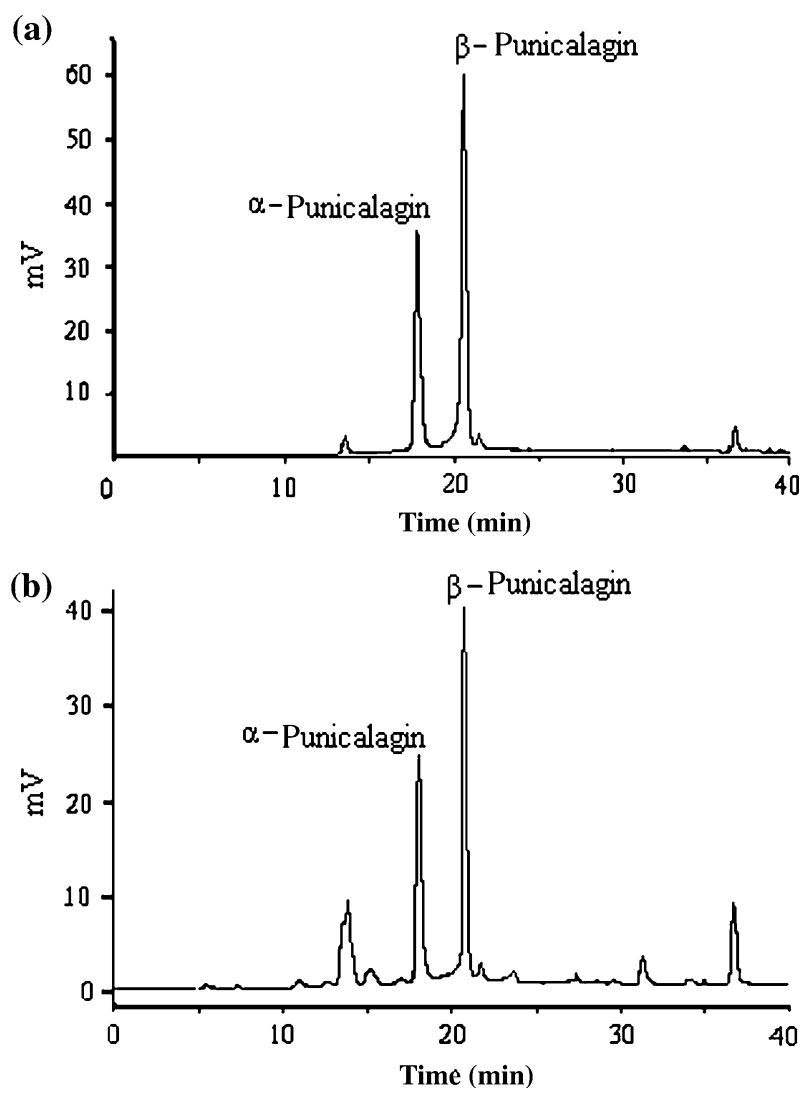

Fig. 1. Chromatograms of the LC elution profile of punicalagin standard (a) and that from pomegranate husk sample (b)

pearl HW 240C column [6]. Reported methods gave the content of punicalagin in extracted samples, but not the real content in pomegranate husk.

A rapid, simple and accurate analytical method is reported here to determine the content of each punicalagin isomer and total content of punicalagins in pomegranate husk. Punicalagins in 16 varieties of pomegranate husk were measured.

\section{Experimental}

\section{Sampling}

Fourteen varieties of fresh pomegranate husk were collected from seven provinces in China and two varieties of dried pomegranate husk were from drugstores. Punicalagin isomer standards were obtained by HSCCC as we reported before [8]. Ethanol used for sample preparation was of analytical grade and purchased from Beijing Chemical Factory (Beijing, China). Trifluoroacetic acid (TFA) used for LC analysis was of chromatography grade and purchased from Merck (Hohenbrunn, Germany). Methanol used for LC analysis was of chromatography grade and purchased from Dima (Dima Techonlogy Inc., USA).

\section{Sample preparation}

Fresh pomegranate husk was dried naturally at room temperature then grounded to powder. One gram of dried husk powder was extracted ultrasonically with $30 \mathrm{~mL} \mathrm{40 \%} \mathrm{ethanol} \mathrm{for} 30 \mathrm{~min}$ twice. The supernatants were combinded, ethanol was evaporated under vacuum. The extract was freeze-dried, yielding about $0.5 \mathrm{~g}$ crude sample. Thirty milligram of the sample were precisely weighed by a Sartorius scale (BP211D) and dissolved in a $10 \mathrm{~mL}$ volumetric flask with methanol-water $(1: 1)$. Then it was filtered through a $0.22 \mu \mathrm{m}$ membrane filter prior to injection.

\section{Operating Conditions}

The LC used was a Shimadzu LC-20AVP system with two LC-20AT solvent delivery units, an SPD-20A UV-vis detector, a CTO-10ASVP column oven (Shimadzu, Kyoto, Japan), a T2000P workstation (Beijing, China) and a reversed phase $\mathrm{C}_{18}$ column $(250 \times 4.6 \mathrm{~mm}$, $5 \mu \mathrm{m}$, Diamodsil ${ }^{\mathrm{TM}}$ ).

The solvent system consisted of $\mathrm{MeOH}$ (eluent A) and $0.1 \%(v / v)$ TFA in water (eluent B). Gradient conditions: 0$10 \mathrm{~min}, 5-20 \%$ A in $\mathrm{B} ; 10-25 \mathrm{~min}, 20-$ $45 \% \mathrm{~A}$ in $\mathrm{B} ; 25-35 \mathrm{~min}, 45-65 \% \mathrm{~A}$ in $\mathrm{B}$; $35-38 \min 65 \% \mathrm{~A}$ in $\mathrm{B}$. This was followed by a $10 \mathrm{~min}$ re-equilibration. The column oven temperature was set at $30{ }^{\circ} \mathrm{C}$. The flow rate was $1.0 \mathrm{~mL} \mathrm{~min}{ }^{-1}$, and $5 \mu$ portions were injected into the column. Punicalagin was detected by absorbance at $378 \mathrm{~nm}$. The representative chromatograms of the LC elution profile of punicalagin standard and that from pomegranate husk sample is shown in Fig. 1.

\section{Preparation of the Standard Curve}

Quantification was based on the external standard method. A stock solution was prepared with $10.0 \mathrm{mg}$ of punicalagin reference standard, which was dissolved and diluted to $5 \mathrm{~mL}$ with methanolwater $(1: 1) .2,3,4,5,6,8 \mu \mathrm{L}$ were injected in duplicate. Peak areas were recorded for all the solutions.

\section{Statistical Analysis}

All data of total punicalagin content are presented as mean + standard deviations (SD). The equilibrium constant $K$ of $\beta$-punicalagin and $\alpha$-punicalagin content was also calculated. It was defined as $\beta$-punicalagin content divided by $\alpha$-punicalagin content. Standards and samples of each variety of pomegranate husks were dissolved in methanol, water and methanol-water 1:1 solution, respectively. 


\section{Results and Discussion}

\section{Linearity of Standard}

Linear regression analysis of the peak area responses $(y)$ versus the injection volume $(x)$ gave the following equations: $y=137746 x+141648$ for $\alpha$-punicalagin, $y=224830 x+167999$ for $\beta$-punicalagin and $y=362576 x+309648$ for total punicalagins. The correlation coefficients $\left(r^{2}\right)$ were $0.9983,0.9995$ and 0.9998, respectively, which demonstrated linearity of the method over the concentration range analyzed. Three correlation coefficients had significant differences after verification $\left(r_{0.05}(4)=\right.$ $0.811 ; r_{0.01}(4)=0.917$ [9]).

\section{Precision and Recoveries}

The system precision was determined by chromatographing six injections of the standard solution and calculating the relative standard deviation (RSD) of the peak area responses. The method precision was established by assaying six different extracts of the same husk sample with the proposed chromatographic method. The RSD for standard and samples were 1.05 and $0.74 \%$, respectively. In our study, recovery is related to extraction and quantification of punicalagin after the autolysis. Triplicate analyses of a husk sample following the addition of known amounts of punicalagin standard provided recoveries (mean \pm RSD) for punicalagin of $98.1 \pm 0.94 \%$.

\section{Analysis of Punicalagin Content in Pomegranate Husk}

The LC method was used to quantitate punicalagin in a sample of dried pomegranate husk. Since pomegranate-derived foods are widely consumed and there have been conflicting reports regarding the toxicity of punicalagin $[10$, 11], there is a need for in depth in vitro and in vivo studies to determine the biological properties of this compound. Thus, it is important to analyze the content of punicalagin in the traditional

Table 1. Total punicalagin content in various pomegranate husk

\begin{tabular}{|llc|}
\hline Pomegranate husk accession & $\begin{array}{c}\text { Total content of punicalagins } \\
\text { in husk }\left(\mathrm{mg} \mathrm{g}^{-1}\right)\end{array}$ \\
\hline Province & Area or name of variety & \\
\hline Xinjiang & Black husk & $56.5 \pm 0.8$ \\
Shangdong & Binzhou & $80.5 \pm 1.3$ \\
Shanxi & Lintong & $66.0 \pm 0.3$ \\
Sichuan & Panzhihua & $82.7 \pm 0.1$ \\
Sichuan & Huili & $80.3 \pm 0.1$ \\
Shanxi & Fengxiang & $75.9 \pm 0.3$ \\
Yunnan & Dali & $80.8 \pm 0.5$ \\
Anhui & Huaiyuan & $89.8 \pm 0.1$ \\
Xinjiang & White husk & $92.5 \pm 0.8$ \\
Hunan & Changsha & $44.9 \pm 0.2$ \\
Yunnan & Mengzi & $47.8 \pm 0.8$ \\
Shangdong & Taishan & $97.6 \pm 0.6$ \\
Shanxi & Red ruby & $121.5 \pm 0.2$ \\
Western & Soft seed & $11.0 \pm 0.9$ \\
Heibei & Drug store & $97.3 \pm 0.8$ \\
Guangdong & Drug store & $39.8 \pm 0.3$ \\
Mean & & 82.4 \\
\hline
\end{tabular}

Values represent mean $\pm \mathrm{SD}, n=3$

Chinese medicine-pomegranate husk. Table 1 shows the total content (triplicate analyses) of punicalagins of 16 different varieties of pomegranate husk. The highest content of punicalagins in husk was found in the husk of a pomegranate named red ruby from Shanxi province $\left(121.5 \mathrm{mg} \mathrm{g}^{-1}\right)$, which is nearly threefold the lowest content in husk from a drugstore in Guangdong province $\left(39.8 \mathrm{mg} \mathrm{g}^{-1}\right)$. Data showed significant differences in the content of punicalagins among husks from various areas.

\section{Equilibrium Constant of Punicalagin Content in Pomegranate Husk}

It is reported that $\alpha$-punicalagin and $\beta$-punicalagin interconverted and the equilibrium constant, $K=[\beta] /[\alpha]$ was one in methanol and four in water [11]. But in our study, the ratio of $\beta$-punicalagin and $\alpha$-punicalagin content was about 1.60. It is interesting to note that this ratio will not change with the change of solvent.

\section{Conclusion}

Punicalagin, which mainly exists in pomegranate fruits, such as pomegran- ate husk, pith and carpellary membrane [12], is of great interest because of its antioxidant activities. However, in our studies the punicalagin levels have shown to be different among various accessions of pomegranate husk. It is very important to select several varieties with high punicalagin levels from numerous pomegranate husk accessions in drugstores or supermarkets for the study. The described method was found to be rapid, linear, reproducible, specific, accurate and capable of analyzing a large number of samples without elaborate sample preparation.

\section{Acknowledgments}

The authors acknowledge the support of the Natural Science Foundation of China (20576010) and the Program for New Century Excellent Talents (NCET-050117).

\section{Open Access}

This article is distributed under the terms of the Creative Commons Attribution Noncommercial License which permits any noncommercial use, distribution, and reproduction in any medium, provided the original author(s) and source are credited. 


\section{References}

1. Lei F, Xing DM, Xiang L, Zhao YN, Wang W, Zhang LJ, Du LJ (2003) J Chromatogr B 796:189-194. doi:10.1016/ S1570-0232(03)00610-X

2. Kulkarni AP, Mahal HS, Kapoor S, Aradhya SM (2007) J Agric Food Chem 55:1491-1500. doi:10.1021/jf0626720

3. Seeram NP, Lee R, Heber D (2004) Clin Chim Acta 348:63-68. doi:10.1016/ j.ccen.2004.04.029

4. Seeram NP, Lee R, Hardy M, Heber D (2005) Sep Purif Technol 41:49-55. doi: 10.1016/j.seppur.2004.04.003
5. Mertens-Talcott SU, Jilma-Stohlawtz P, Rios J, Hinorani L, Derendorf H (2006) J Agric Food Chem 54:8956-8961. doi: 10.1021/jf061674h

6. Li HX, Zhang HL, Liu YZ, Du J (2006) Chin Tradit Herb Drugs 37:780-782

7. Tzuker R, Glazer I, Bar-Ilan I, Holland D, Aviram M, Amir R (2007) J Agric Food Chem 55:9559-9570. doi:10.1021/ jf071413n

8. Lu JJ, Wei Y, Yuan QP (2007) J Chromatogr B 857:175-179. doi:10.1016 j.jchromb.2007.06.038

9. Wang QD, Yang J (2003) In: Wang DP, Yang S, Liu L (eds) Food experiment design and data treatment. China Agriculture University Press, Beijing, p 481

10. Scalbert A, Morand C, Manach C, Cemesy C (2002) Biomed Pharmacother 56:276-282. doi:10.1016/S0753-3322(02) 00205-6

11. Doig AJ, Williams DH, Oelrichs PB, Baczynskyj L (1990) J Chem Soc Perkin Trans 1:2317-2321. doi:10.1039/ p19900002317

12. Kulkarni AP, Aradhya SM, Divakar S (2004) Food Chem 87:551-557. doi: 10.1016/j.foodchem.2004.01.006 